\title{
The Deep Self: An Analysis of Art Brut Symbols Based on Individual Unconsciousness
}

\begin{abstract}
Yu Fan.
School of Fine Arts, Hunan normal University, Yuelu District, Changsha City, Hunan Province, China. 1832926983@qq.com.

ABSTRACT

Based on the individual unconsciousness proposed by psychologist Carl Jung, this paper makes a picture analysis of the Art Brut works created by prisoners. On the one hand, it focuses on the symbols generated by creative association in the process of meaning generation of national culture. On the other hand, the author's life experience is combined to explore the individual unconsciousness and complex conveyed by the symbols of his works. Through the study of symbols, this paper aims to dig out the strong primitive power of human beings contained in the original works of art, which is called irrational power, but is exactly missing in modern civilization.
\end{abstract}

Keywords: Art Brut, symbolic analysis, individual unconsciousness, art therapy

\section{深处的自我一一基于个体无意识的原生艺术符号分析}

\author{
余凡
}

湖南师范大学美术学院美术学, 岳麓, 长沙, 湖南, 中国 1832926983@qq.com

\begin{abstract}
摘要
本文基于心理学家荣格提出的个体无意识，对服刑人员创作的原生艺术作品进行画面分析。一方面，关注民族 文化在意义生成过程中由于创造性关联产生的符号; 另一方面, 结合创作者的生平经历, 探究其作品符号所传 达的个体无意识与情结。本文旨在通过符号的研究, 挖掘原生艺术作品中蕴含着强烈的人类原始力量, 这些被 称为非理性的力量, 恰恰为现代文明所缺失。
\end{abstract}

关键词：原生艺术；符号分析；个体无意识；艺术治疗

\section{1.前言}

原生艺术为二战后(1945 年)被发掘的“非正统” 艺术种类, 由法国艺术家让·杜布菲发现于瑞士精神 病院中病人的创作活动, 原生艺术的起源与精神障碍 息息相关, 在长达几十年的发展中, 原生艺术的创作 群体由精神病患者扩展至通灵者, 具有高度颠覆性与 边缘倾向的民间自学者 ${ }^{[1]}$ 。本文中研究的作品由服刑 人员所创作, 作者文化程度普遍不高, 相当一部分因 其精神障碍而犯罪入狱, 或是在狱中长期服刑, 与社 会割裂。早在 1921 年, 瑞士精神病学家沃尔特 - 摩 根泰勒就发表了一本专著讲述精神分裂患者的人生 和艺术作品, 通过视觉图式将精神病患者的世界展现 于众。本文将通过分析四位服刑人员的五幅作品, 探
究这些极少受到现代文明浸染的创作者以何种方式 表达自己, 以及表达了怎样的潜意识, 关注原生艺术 作品中的个人价值与社会意义。

\section{2. 原生艺术中的个体无意识}

精神分析心理学家荣格荣格把无意识区分为个 体无意识和集体无意识两部分。个体无意识是潜藏在 意识层次之下的一个较薄的层面,邻近 “自我”。个人 无意识如同一个容器, 与有意识的个体化机能不相一 致的心灵活动和曾经一时的意识经验 ${ }^{[2]}$, 如果受到忽 视或者积压，都会被储藏在个体无意识中，如果其继 续与人格中心特征背离, 继而将分裂成自我情结。原 生艺术创作群体往往因其精神障碍或人生经历有着 不同程度的分裂情结，不论其清醒、犯罪后的醒悟， 
还是在服刑期间受到规训后，其意识中曾经不一致的 心灵活动和一时的意识经验都在不断地背离现在的 认知意识, 并与之拉开距离, 产生矛盾。就原生艺术 创作群体而言，其艺术创作没有过多文化符号的介入， 作品符号表达与其个体无意识紧密相连。结合创作者 的人生经历可帮助探究创作者个体无意识中可能存 在的某些情结, 为艺术治疗提供基础。

\section{3.个体无意识符号分析}

\section{1 “人-物” 合一符号}

在分析原生艺术画作的过程中，画面中含有人与 动植物融为一体的画面符号占据本文考究画作的近 百分之十, 画面符号不是单纯地将物拟人化, 而是将 人的形象与自然界中的其他物象进行一定的融合, 并 构建为一种新的形象。“人-物” 合一这一符号的所指 为一一希望通过绘画表现的融合状态达到人物与其 所认知的物象属性合一。在对原生作品符号分析时, 不仅要注意符号在原始与传统文化中的象征含义, 还 要结合作者的所思所想所经历的社会生活具体分析, 以挖掘其隐匿的情结。图 1 和图 2 是一位复发性抑郁 障碍男性患者阿伟所作, 创作者文化程度不高, 但因 抑郁障碍学过几个月绘画, 因此具备对物象有一定的 表达能力。在阿伟的自我描述中, 妻子因其入狱而主 动离开, 他对妻子充满愧疚和思念, 也希望妻子生活 的快乐健康 ${ }^{[3]}$ 。

这两幅作品都出现同样的绘画符号: 女人体、荷 叶状及藕状几何图案以及波状线条。首先被符号化的 是莲花与鱼, 一般来说, 鱼象征着繁殖, 尤其在中国 母亲氏族社会遗址出土的陶器上常见鱼纹符号, 这些 符号是女性的生殖象征, 鱼纹等相关的简化符号, 有 着女阴崇拜的内涵, 画面中的人物符号与动物符号息 息相关。莲花的原始意向是生殖女神, 莲花形似女阴, 莲蓬使人联想到繁殖, 因此即使是现代, 也会用莲花 来比喻美女。而当鱼和莲同时存在时, 鱼在莲的根茎 部环绕, 常用来比作男女之欢。作者将妻子的头发画 成荷叶状, 因此不难理解作者在画中, 把妻子比作莲 花, 在画面的下半部分画满鱼或者鱼波纹符号, 是作 者将自我意识投射至有关于鱼的符号中。在图 1 与图 2 中, 作者用荷叶与鱼的关系, 象征对妻子的庇护, 是作者保护欲的体现。在图 1 中, 同样出现的几何符 号一一画面左边的线条是鱼波纹, 是相关的女性生殖 崇拜, 而右边是直线描绘, 象征着所处的环境, 是一 根根坚硬的围栏。在空间上, 画面分为前后两个层次, 画面背景是一连串以莲藕为原型的几何符号构成的 网状环形, 颇有被束缚的意味。而面向观者的画面是 女人体以及密集的鱼, 象征着家庭、妻子与自由, 整 个前后画面处于一张网中, 也意味的现实空间上的束 缚。

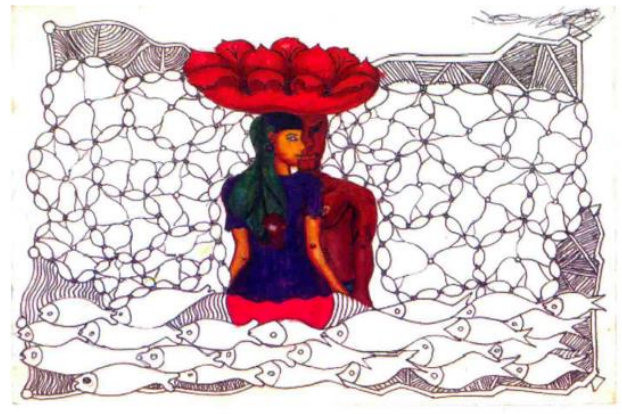

图 1 《美之化身》 阿伟

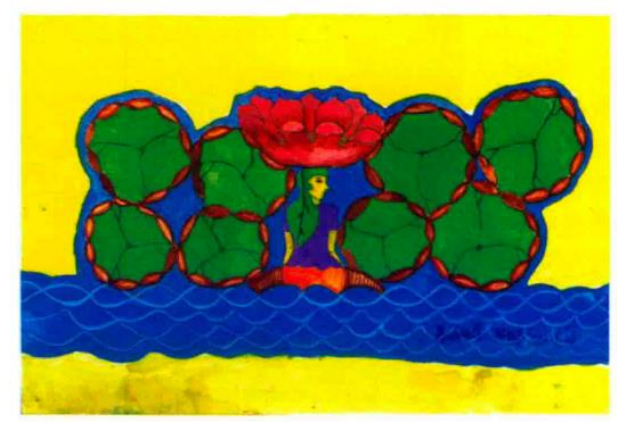

图 2 《美的化身》 阿伟

除了通过 “他者” 来表达无意识外, 原生艺术创 作者还会直接通过自身描绘以表达深藏的潜意识。如 在所研究的一幅作品中, 有一幅将自己的脸画在龟身 的画作, 达到人的 “兽我” 层次。虽然不了解作者在 服刑前的经历, 但不妨结合中国传统的龟文化, 探究 其中的无意识表达。龟在我国传统文化观念中为神物, 《礼记》中也有 “麟、风、龙、龟谓之四灵” 的相关 记载。特别在原始时代, 有着明显的龟崇拜。原始祖 先发现, 龟行动缓慢, 活得时间比一般动物要长, 因 此龟至隋唐时期之前, 都是长寿的象征, 如汉代朝廷 把龟同帝王和皇权直接融于一体, 把龟与皇族的祖宗 供奉在一起, 以保汉室江山千秋万代。烧裂的龟甲也 是可以用来占卜的神物, 这种传统文化象征, 在历史 的流传中形成了一定的集体无意识, 因此在许多影视 中可见, “龟” 是 “智者” “大师” 的象征。而经历史 记载, 至元代, 龟文化开始变异, 龟不再是象征灵威 的神物, 而是伤风败俗之物, 如市井中常用 “龟儿子”

“王八龟” 这样含有 “龟” 的词语形容坏人, 或者他 人的弱点。正因为龟文化在发展过程中的变异, 流传 下来, 形成了 “龟” 的象征的两极性, 因此在作者将 自己的脸与龟身结合时, 可能是表达对活得长久的渴 望, 亦可能是对自己犯罪的人格诠释。

\section{2 女性符号}

在原生艺术作品中不难发现, 几乎所有男性作者 选取的描绘对象都是女人体或者相关的女性符号。在 符号学的研究中, 女性符号曾是各个领域的焦点, 早 期在男权社会, 视觉图式中的女性符号通常是男性目 光过滤后的产物, 象征男性的性渴望 ${ }^{[4]}$ 。在原生艺术 中, 通常也是如此, 不同的是, 原生艺术除了给予画 
面中女性符号以性想象, 也将女性视为人生道路上的 救赎。图 3 是一位男服刑人员所作, 名为《走过的路》, 作者在创作此作品时, 已服刑多年。从画面结构上看, 一位半裸的女人仁立在田野里向远方眺望, 由女人外 衣引申出的路指向远处的山丘和湖泊, 路边的田野里 生出一颗茂密的树。画面中的女性是作者心中美好事 物的无意识表征, 亦是作者意识的投影: 自我消解与 救赎。作者将自我完成的心理意识投射至画面中的女 人体, 女人的衣服在分解, 解离的衣服延伸出一条路, 消融在远处的山河。解离的过程象征着作者自我救赎 的过程, 而远处开阔的地点与意向, 则是自我完成的 标志, 与此同时, 女性裸体亦是自我完成结果在画面 中的符号表示。作者将异性符号化以承载自我救赎的 无意识, 其一可能来源于性经历 (包括与异性的亲密 交往）或是他人传达的经验中对女性的印象; 其二很 可能是将自己的母亲或是对母亲的认知投射至画面 中的女性符号。值得注意的是, 从画面整体来看, 路 边的树略显突兀, 很可能象征与异性相处过程中的某 些回忆内容, 或是与母亲、家庭相处过程中对自己影 响较大的事件与记忆, 树作为一个事件的符号化象征 而存在。

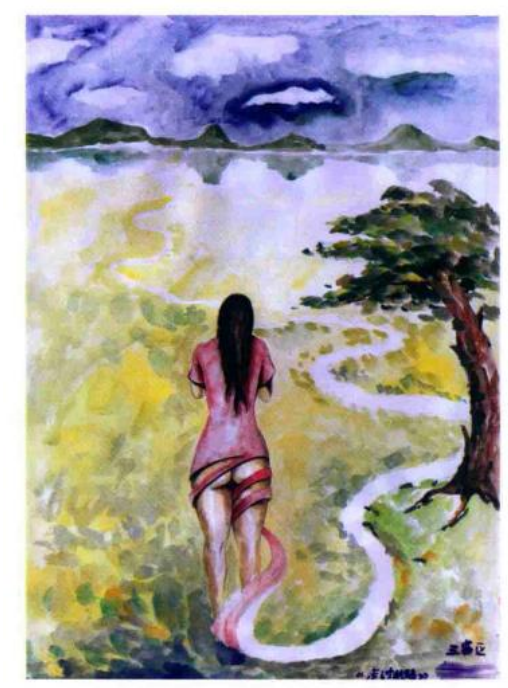

图 3 《走过的路》阿忠

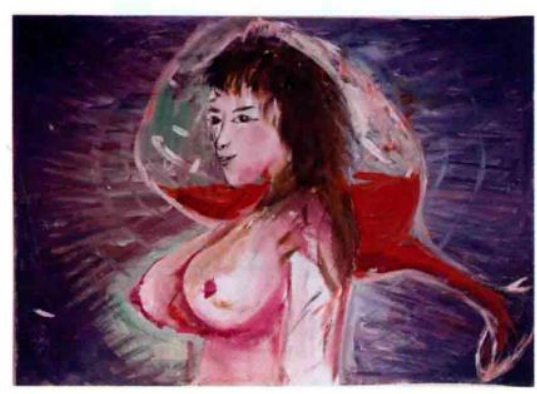

图 4 《梦中人》 阿标

原生艺术创作中, 有不少直接描绘女性裸体与性 器官的作品, 如图 4, 女性特征被符号化, 符号的所 指更多地是创作者对于异性的想象以及对性的渴望。 对于精神患者和高度社会边缘化的服刑人员来说, 这
些象征符号是对原欲能量的宣泄。在传统艺术中, 与 性相关的符号通常被视为不雅之作，而在原生艺术中， 相关的创作是他们对原欲的宣泄途径, 亦是能量的转 移, 通过这种转移, 降解自身的犯罪欲望, 消解造成 有害行为的相关情结。

\section{3 人脸符号}

图 5 是一位精神障碍患者画的人脸肖像, 图中左 上角文字表明这是一幅精神分裂者的脸部肖像。这幅 图呈现出一张极为复杂的、不完整的人脸, 其中不乏 恐怖元素, 甚至有些论异。但是在这张画像中, 藏有 许多精神分裂患者的人格特质。首先, 画面的右边整 体色调是橙红色, 这样的颜色使得右脸呈现出暴怒状 态, 是精神分裂患者常见的一面一一感性、好发脾气、 无端恐惧。且不难发现, 右边头上的锯齿角比左边的 更为粗长, 意味着比左脸更为愤怒激进的精神状态, 不仅如此, 额头上的皱纹是直线勾勒, 皱纹下面是翻 滚的眼珠, 眼下黑色用色面积更大, 耳朵里生出刺, 鼻毛挺立, 牛角从嘴里突出, 连脖子也是红色的。这 些锯齿、直线、颜色符号, 无不暗示着右脸是一个具 有杀伤力, 且恐惧的人格状态。实际上精神分裂患者 的一面状态与右脸种种符号表达出意味是相似的, 精 神分裂者伴有精神和人格分裂, 其中一面表现为性格 反常, 好无故发脾气, 不能自制, 或无端恐惧 ${ }^{[5]}$ 。

而另一面人格藏在画面的左边。这是一个更为阴 郁、阴冷怪癖的状态一从画面的整体紫色色调看来。 齿状皱纹与眉形暗喻着左边为自我保护状态, 防御性 极强, 与右边翻滚的眼珠不同, 左边的眼睛好似一幅 深不见底的山水画, 情感更为复杂深遂, 嘴里生出的 不是牛角, 而是更为软弱的类似于毛毛虫的物体。除 了暴怒的一面, 精神分裂患者的另一面敏感多疑, 沉 湎于一些脱离现实的幻想、自语、自笑, 在实际行为 中患者精神活动变得迟针, 对人冷淡, 与人疏远, 身朵 避亲人并怀敌意。左边阴郁的色调, 紧皱的眉头, 肥 大而略显紧张的耳部描绘, 似乎都是另一面人格的真 实写照。从画面整体看来, 左边脸部描绘比右边的曲 线运用更多, 在线条符号方面, 直线是感性状态的符 号表达, 如嘴边牛角与直线, 有直言顶撞的意味; 曲 线多为理性状态的情感表达, 尤其是在本幅画面中, 暗示着作者多处于理性的防御状态。如果观察仔细, 还能发现画面中的人物处于极度悲痛的状态——两 侧脸上均有雉状泪珠滑落, 但是左边的雉状符号明显 比右边多, 这种不对称性的物象表现, 也许是作者在 处于精神分裂状态的理性防御人格状态时, 感到更痛 苦的缘故。

如果说画面的种种符号多为作者个体的无意识 表达, 那么参杂着意识与无意识的符号, 即为将这张 有着双面人格象征的人脸䢃开的 “双色箭”。“箭” 的 图符能指是一支带有杀伤力的尖状物, 所指是攻击、 是对物体或者现状的破坏。此图中, 箭符意味着作者 在受到规训后的自我意识, 或者是外部力量, 这个自 我意识或者外部力量, 将身体所呈现的两种人格粗暴 
地分开，从根源上将人格摧毁。整幅画作，是作者对 自身疾病认知和态度的无意识体现。

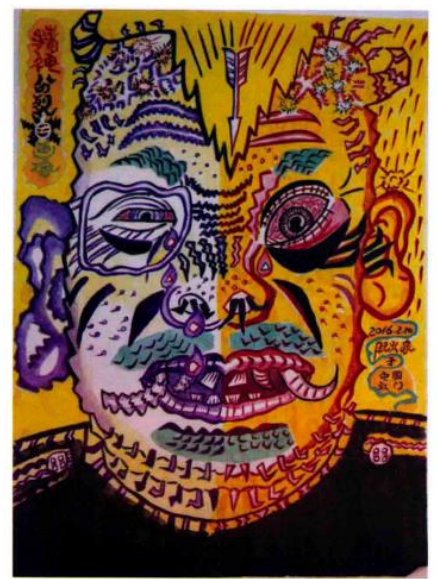

图 5 《精神分裂》 阿泉

\section{4.结语}

不论是个体无意识生发出的情结, 还是集体本能 无意识的原欲力量, 在实现受阻时需要一定的能量转 移。原生艺术创作活动通常承载着创作者的直接情感 输出, 符号的所指是其在生成过程中相关的个人或社 会意义，因此这些看似与主流艺术相异的创作，在一 定程度上是现代文明所压抑的非理性输出, 也是人类 最接近自然的力量。

\section{REFERENCES}

[1] Kelly, M. (1998) Encyclopedia of Aesthetics,2d. Oxford university Press. New York.

[2] Yin, L. (2002) A brief introduction of mind structure in analytic psychology of conscious individual unconsciousness and collective unconsciousness. Social Science Research,[J]. 2002, (2): 62-65.

[3] Qiu, H. (2018) Native art and psychotherapy. Jinan University Press, Guangzhou.

[4] Li, S. (2000) Female symbols in Advertising: A Cross-cultural Comparison. Journal of Southwest Minzu University(Humanities and Social Science), [J]. 21: 75-78.

[5] Chen, Z. (2015) Medical Genetics. People's sanitary publishing press, Beijing. 